\title{
Statyba
}

\section{THE RELATIONSHIP BETWEEN THE CRITERIA OF MULTIPURPOSE SELECTION AND THE CHARACTERISTICS OF RELIABILITY}

\section{E. K. Zavadskas}

To cite this article: E. K. Zavadskas (1996) THE RELATIONSHIP BETWEEN THE CRITERIA OF MULTIPURPOSE SELECTION AND THE CHARACTERISTICS OF RELIABILITY, Statyba, 2:6, 23-26, DOI: $10.1080 / 13921525.1996 .10531640$

To link to this article: https://doi.org/10.1080/13921525.1996.10531640

Published online: 26 Jul 2012.

Submit your article to this journal $\llbracket$

Џ Article views: 33

4 Citing articles: 1 View citing articles 준 


\section{ZUSAMMENHANG ZWISCHEN DER MEHRZWECKSELEKTIERUNG UND DER ZUVERLÄSSIGKEIT IM BAUWESEN}

\section{E.K.Zavadskas}

\section{Eiulcitung}

Als Ergebnis der Mehrzweckselektierung (MS) $[1,2,3]$ - der Auswahlzwecks des Ersatzes und der Erncuerung - ist die Wahl der rationellen Projekt und technologischen Lösung, die auf die Erarbeitung oder Auswahl aus der etwaigen Menge der möglichen Varianten des bestimmten funktionierenden technischen oder organisatorisch-technologischen Systems gerichtet ist.

Alle Berechnungen, die bei der MS durchgeführt werden, können in apriori und aposteriori unterschieden werden. Die aprioren Berechnungen werden im Stadium der Projektierung von Gebäuden und Bauwerken sowie bei der Erarbeitung der Projekte der Organisation der Bauensführung und der Projekte der Durchführung der Arbeiten mit der Anwendung in den Berechnungen der normativen Materialien und der Empfehlungen zur wahrscheinlichen-statistischen Bewertung der Parameter und Charakteristiken vorgenommen. Aposteriore Berechnungen werden mit der Berücksichtigung der konkreten Informationsmaterialien durchgeführt, die an Hand der Durchführung der statistischen Bearbeitung der Ergebnisse der experimentellen Untersuchungen, natürlichen Beobachtungen, chronometrischen Bemessungen in der Abhängigkeit von der Art der zu lösenden Aufgabe erhalten waren.

Die Rationalität der Funktionierung einer oder anderer technischen oder organisatorisch - technologischen Systems wird durch die Kriterien der MS kennzeichnet, die im endgültigen Ergebnis die Nützlichkeit der vorgeschlagenen Lösung widerspiegeln.

An die Funktionierung beliebigen Systems werden zugleich mit den Anforderungen an seine
Nützlichkeit bestimmte Anforderungen über die Zuverläßigkeit gestellt, die wesentlich von der Art des Systems abhängen. Deshalb entsteht völlig begründet die Frage über den Zusammenhang der Begriffe der Nützlichkeit $[4,5]$ und der Zuverläßigkeit [6-14].

\section{Zusammenhang zwischen der Mehrzweckselektie- rung und der Kennzeichnungen der Zuverläßigkeit}

Alle technischen und organisatorisch technologischen Systeme werden gewöhnlich in zwei Klassen geteilt: die Systeme der einmaligen (S.d.e.F) und mehrmaligen (S.d.m.F) Funktionierung.

Unter S.d.e.F wird ein System verstanden, daß nach einmaliger Erfüllung seiner Funktion sein Bestehen beendet. Für Zuverläßigkeitskriterium des S.d.e.F wird gevöhnlich die Wahrscheinlichkeit $p_{0}$ der störungslosen Arbeit wahrend des Zeitzyklus der Funktionierung $(t)$ genommen, d.h. $p_{0}(t)$. Dabei kann die Wahrscheinlichkeit der unversaglichen Arbeit nicht immer als Multiplikator in die Nutzensfunktion $[4,5]$ eingeführt werden, weil im Ergebnis der Wert der mathematischen Erwartung der Nützlichkeit erhalten wird, der keinen Sinn beim einmaligen Prozeß hat. Die Einführung $p_{0}(t)$ als Multiplikator bei der Bestimmung der Nutzensfunktion kann zum Ersatz der ungenügenden Zuverläßigkeit als Überschuß in die Werte anderer polidimensionaler Effektivitätskennziffern spezifischer Kriterien der Optimalitat (p.E.K) führen, dic bei der Ermittlung der Kriterien der MS berücksichtigt werden.

Auf solche Weise muß bei MS für S.d.e.F die Wahrscheinlichkeit der Störungslosen Arbeit $p_{0}(t)$ als Begrenzungsbedingung (als Kennziffer- $\Lambda$ nforderung in der Entscheidungsmatrix $\bar{P}$ ) [1] auftreten. 
Dabei konnen in die Iintscheidungsmatrix nur die Varianten einbezogen werden, die die Anforderungen der Wahrscheinlichkeit der störungslosen Arbeit crfüllen. Zum Beispicl sind in den Arbeiten vom A.G. Roitmann [15] die normativen Werte $p_{0}(t)$ in der Abhängigkeit von der $\wedge$ rt der Konstruktionen gegeben, die 0,85 für die selbstlragende raumumschließende Elemente, 0,95 für die Elemente der statisch unbestimmten Systeme, deren Versagen in der Festigkeit die plötzliche Zerstörung des Systems bewirkt, 0,99 für tragende Elemente mit den stufenweisen Absagen (der Decken, Pfeiler, Binder); 0,999 für die verantworliche Konstruktionen mit den plötzlichen Absagen betragen. Dabei wird allgemein die Konstruktion als System betrachtet, wenn sie wiederaufgebaut wird, und als Element, wenn sie nicht wiederaufgebaut wird. Wenn die MS der technischen Lösungen für die erwähnten Konstruktionslösungen vorgenommen wird, so werden dic Varianten, die die aufgezählten normativen Werte nicht erfüllen, in die Entscheidungsmatrix nicht aufgenommen. Die Kennziffer $p_{0}(t)$ wird dabei in die Matrix $\bar{P}$ [1] nicht cingcführt.

Jedoch kann bei der massenhaften Anwendung des S.J.e.F die Kennziffer $p_{0}(t)$ in die Entscheidungsmatrix als eine p.E.K eingeführt werden. Als p.E.K bei der Bestimmung der Nutzensfunktion oder der Kriterien den MS der Projekte und der technologischen Lösungen der Bauproduktion kann dic Kennziffer $p_{0}(t)$ auch fur die Fälle angenommen werden, wenn das Versagen mit dem Unfall nicht droht.

Auf solche Weise wird S.d.e.W bei der MS nach den Kriterien eingeschätzt, die den Komplex der p.E.K berücksichtigen, in deren Bestand auch die p.E.K eingehen können, die die Zuverläßigkeit des Systems kennzeichnen.

Wenn dic Variante $B_{i}(i=\overline{l, m})$ [1] durch p.E.K $\bar{x}_{j}(j=\overline{l, n}) \quad[1]$ charakterisiert wird, so kann zusammen mit den p.E.K, die die anderen Zwecke kennzeichnen, zu deren Erziclung der Fachmann bei der MS $\bar{x}_{i j}$ strebt, in die Entscheidungsmatrix $\bar{P}$ auch die p.E.K eingen, dic die Zuverläßigkeit der
Varianten charakterisiert. In der Abhängigkeit von der Art der zu lösenden Aufgabe der MS kann p.E.K verschiedene Wichtigkeit haben. Dabei sind folgende extreme Fälle:

1) die Bewertung der Zuverläßigkeit ist nicht erforderlich oder wird die Bewertung in die Begrenzungen, d.h. in BegrenzungenAnforderungen überführt;

2) wenn die Zuverläßigkcit die primärc Rolle spiclt (zum Beispiel in den Fällen, wenn man auf beträchtliche Vertauerung des Systems zwecks Erlangung sogar unbeträchtlicher Erhöhung seiner Zuverläßigkeit gehen darf).

Wenn wir in der Entscheidungsmatrix $\bar{P}=\left[\bar{x}_{i j}\right]$

[1] dic p.E.K einschreiben, die die Zuverläßigkeit der Varianten $B_{i}$ widerspiegelt, mit dem Wert $x_{l}$, so können die extreme Fälle nach den Vektoren der Wichtigkeit bewertet werden, die entsprechend das Bild

$$
\begin{aligned}
& q^{1}=\left(0, q_{2}, q_{3}, \ldots, q_{j}, \ldots, q_{n}\right), \quad j=\overline{l, n} ; \\
& q^{\Pi}=(1,0,0, \ldots, 0, \ldots, 0), \quad j=\overline{l, n} ;
\end{aligned}
$$

haben.

Im Falle (1) $q$, p.E.K $\bar{x}_{l}[1]$ wird gleich Null angenommen und die Bewertung der Varianten nach $n$-1 p.E.K durchgeführt. Im Falle (2) hauptsächlich bei der massenhaften Anwendung des S.d.e.F und wenn die Zuverläßigkeit die primäre Bedeutung hat, wird der Wert der Wichtigkeit $q_{1}$ p.E.K $\vec{x}_{l}$ gleich eins $q_{1}=1$ angenommen. Es ist zu bemerken, daß in einigen Fällen $\bar{x}_{l}$ [1] die gleiche Wichtigkeit mit den andere p.E.K haben kann oder durch den Fachmann auf Grund seiner Erfahrung oder auf der Basis der Ergebnisse der Expertenuntersuchungen [4] festgelegt werden kann.

S.d.m.W zum Unterschicd vom S.d.e.W wiederholen vielmal den vollen Zyklus ihrer Funktionierung. Im Ergebnis der Erfüllung der vollen Zyklen der Funktionierung können die quantitativen Werte einiger p.E.K als Folge der zufälligen Absagen der einzigen Elemente des Systems schlechter werden (die zu maximisierenden - sich verkleinern, die zu minimisierenden sich vergrößern). Aul solche Weise wird die weitere Funktionicrung des Systems weniger nüızlich oder überhaupt unmöglich. Dabei kann 
jedoch keine Schlubfolgerung gezogen, daß die gesamtc Nützlichkeit während der Funktionierung des Systems fehlt oder minimal ist.

Also ist dic Nützlichkeit der S.d.m.F unter Berücksichtigung der BetriebszuverläBigkeit als mittlerer Nutzen nach allen möglichen Zuständen des Systems 2 zu 4 bestimmen.

Wollen wir einen spezifischen Fall betrachten [13], wenn sich die S.d.m.F nur in zwei Zuständen befinden können: im arbeitsfähigen und im arbeitsunfähigen. Dabei nehmen wir an, daß dic mittlere Zeit der geleisteten Arbeit auf ein zufälligen Versagen $\tau_{0}$ gleich ist und die mittlere Zeit der Zuführung des S.d.m.W zum normalen Zustand nach seinem Versagen $\tau_{l}$ gleich ist, dann kann die mittlere Nutzlichkeit des Systems $\bar{u}(x)$ nach dem Ausdruck

$$
\bar{u}(x)=u_{0}(x) \frac{\bar{\tau}_{0}}{\bar{\tau}_{0}+\bar{\tau}_{l}}+u_{1}(x) \frac{\bar{\tau}}{\bar{\tau}_{0}+\bar{\tau}_{l}},
$$

crmitteltwerden, wo $u_{0}(x)$ die Nützlichkeit des völlig intakt funktionierenden Systems; $u_{l}(x)$ die Nützlichkeit des Systems, in dem das zufällige Versagen eingetreten ist; $\bar{\tau}$ die mittlere Zeit der störungslosen Arbeit des Systems sind.

Vicle S.d.m.F zeichnen sich durch viele Kanäle aus, d.h. sind solche, daß der Ausfall eines paralell arbeiteneden Untersystems zur Beendigung der Funktionierung des Systems nicht führt. Dabei werden nur die Charakteristiken des S.d.m.F kleiner, aber das System als ganze kann durch die mittlere Nützlichkeit $\bar{u}(x)$ :

$$
\bar{u}(x)=\frac{\sum_{l=0}^{v-1} u_{l}(x) \bar{\tau}_{l}}{\tau_{l}+\sum_{l=0}^{v-1} \tau_{l}},
$$

kennzeichnet werden. Hier bedeuten: 0 - die Anzahl der arbeitsfähigen Zustände des Systems, die durch die Nützlichkeiten $u_{l}(x), \quad l=\overline{l, 0}$ charakterisiert werden; $\bar{\tau}_{l}$ - die mittlere Zeit der geleisteten Arbeit in jedem Zustand $l=\overline{1,0} ; \quad \tau_{l}$ - die Zeit der völligen Wiederherstellung des S.d.m.W nach dem Versagen und der Abnahme der Nützlichkeit bis $u_{l}(x)=0$.

Wenn es die Möglichkeit besteht nach den Ausdrücken (3) und (4) dic Werte der mittleren
Nützlichkeit $\bar{u}(x)$ des S.d.e.W zubestimmen, so ist nicht schwer, dic Bevorzugungsreihe der möglichen Varianten unter Berücksichtigung ihrer Nützlichkeit zu bilden:

$$
\begin{aligned}
& \left\{\vec{B}_{i}\right\}=\left\{\bar{u}_{1}(x), \bar{u}_{2}(x), \ldots, u_{i}(x), \ldots, \bar{u}_{m}(x)\right\}, \\
& \left\{\vec{B}_{i}\right\}=\left\{B_{1}, \ldots, B_{m}\right\}
\end{aligned}
$$

für $\forall_{i}=\overline{1, m}$ bei möglichen $(\pi=m !)$ Verstellungen. Im Ergebnis ist die Bildung der Bevorzugungsreihe $\left\{\vec{B}_{i}\right\}$ der MS der möglichen Varianten der S.d.m.F unter Berücksichtigung der Nützlichkeit als beendet zu halten.

\section{Schlußfolgerungen}

Die Rationalität der Funktionierung von technischen und organisatorisch-technologischen Systemen kann durch Kriterien der Mchrzweckselektierung, die dic Nützlichkeit der untersuchten Varianten ausdrücken, bezeichnet werden. Bei der Einschätzung der Nützlichkeit der Variante müßte ihre Zuverlässigkeit beachtet werden. Deshalb wird im Artikel die Methodik dargeboten, die den Zusammenhang der Kriterien der Mehrzweckselektierung und der Kennzeichnungen der Zuverlässigkeit untersucht.

\section{Literatur}

1. E.Zavadskas. Mehrzielselektierung bei der Bauvorbereitung // Statyba, No 3(3), Vilnius: Technika, 1995, p. 58-80.

2. R.Seeling, E.Zavadskas. Mehrzielselektierung bei technologischen Entscheidungen der Bauvorbereitung // IX internationaler Kongress industricles Bauen (IKIB), Leipzig: TH Leipzig, Wiss. Berichte, H.5, 1991, S.170-179.

3. Э.К.Завадскас. Миогоцелеваз сепектоновация технологических решений строительного производства (теоретические основы). Внынюс, 1989. 107 с.

4. E.Zavadskas. Komplexe Bewertung und Auswahl ressourcensparender Entscheidungen im Bauwesen. Vilnius: Mokslas, 1987. 209 S.

5. E.Zavadskas. Variantenauswahl mit der Nutzensfunktion // Wiss. Zeitschrift der TH Leipzig, Ig 14, H.5/6, 1990, S. 263-273.

6. A.Kudzys. Probability estimation of reability and durability of reinforced concrete structures. Vilnius, 1992. $143 \mathrm{p}$

7. К.Капур, Л.Јамбсрсон. Надежность и просктирование систем. Москва: Mıр, $1980.604 \mathrm{c}$. 
8. Maтемaтическе мето) в терии натежіости и эф)ективност: Сиравоник в десяти томах. Том 2 / llo; pex li.B.riencriko. Mockia: Manmoctpocme, $1987.280 \mathrm{c}$

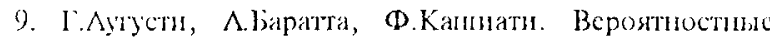
мето:н в етроптелином иросктировании. Москза: Стрийнцат, $1988.584 \mathrm{c}$

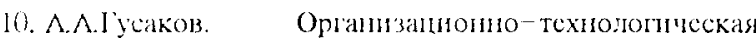

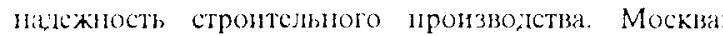
Стройлдат, 1974. $256 \mathrm{c}$.

11. A.II.Kу:зис. Oпсика падежности железобетоних конструктий. Внльнос: Мокспас, 1985. 156 с.

12. E.Borucka, O.Kaplinski. Simulationsmethode zur Bewertung der Zuverlässigkeit von Produktionsprozessen in Plattenwerke // Wiss. Zeitschr. Hochsch. Archit. Bauwes., Weimar, 1982, 2, p. 129-132.

13. Э.К. Завацскас. Выбор рационатылых вариантов комикесной механизации с учстом вероsтностиото характера технологитеских продессов строптельного пропзводства. Вичьнис: ВИСИ, 1985. $36 \mathrm{c}$.

14. І.Балын, э.Завацскас, Ф.ІІедиус. Игровое модепрованис пир подцотовке строителыого произволства. Ленинтрал: ЈІИСИ, 1989. 41 с.

15. АГ.Ройтман. Іандежиость конструкций эксплуа тируемых зданий. Москва: Стройнздат, 1985. $176 \mathrm{c}$.

Iteikta 19960210

\section{IDUGIATIKSLES SELEKTONOVACLJOS IR PATIKIMUMO RYŠYS STATYBOJE}

\section{E.K.Zavadskas}

Santrauka

Technincs ir organizacines technologines sistemos funkcionavimo racionalumas gali būti apibūdinamas daugiatikslès selcktonovacijos kriterijais, kurie išreiškia nagrinejamy̨ variantų naudingumą. Ivertinant varianto naudingumą svarbu atsižvelgti ir i jo patikimumą. Todẻ straipsnyje pateikta metodika, nagrinejanti daugiatikslès selektonovacijos kriterijy ir patikimumo charakteristiky priklausomybę.

\section{TIL REIATIONSIHIP BEIWEEN THE CRTEERIA OF MULTIPURPOSE SELECTION AND TIE CIIARACTERISTICS OF RELIABILITY}

\section{E.K.Zavadskas}

Su in mary

The rationality of functioning of either the technical or the organizing-technological system is characterized by the criteria of multipurpose selection that express the usefulncss of the variants under consideration. When evaluating the usefulness of a variant, it is necessary to take into consideration its reliability. Therefore, the given method investigates into the relationship between the critcria of multipurpose selection and the characteristics of reliability.

Edmundas Kazimieras ZAVAISKAS. Doctor Habil, Professor. Rector of Vilnius Technical University. Member of Lithuanian Academy of Sciences. Member of Ukrainian Academy of Technological Cybernatics. Vilnius Technical University, 11 Sauletekio Ave, 2054 Vilnius, Lithuania.

In $1973 \mathrm{Dr}$ degree in building structures. Assistent, senior assistent, associated professor, professor at the Department of Building Technology and Management. In 1987 Dr Habil degree at Moscow Civil Engineering Institute (problems of building technology and management). Research visits to Moscow Civil Engineering Institute, Leipzig and Aachen Higher Technical Schools. He maintains close academic links with the universities of Aalborg (Denmark), Salford and Glamorgan (Great Britain), Poznan University of Technology (Poland), Leipzig Higher School of Technology, Economics and Culture (Germany) and Aachen Higher Technical School (Germany). Member of international organizations. Member of organizational and programme committees of many international conferences. Member of editorial boards of some research journals. Author of monographs in Lithuanian, English, German and Russian. Research interests: building technology and management, decision-making theory, automation in design, expert systems. 